University of Nebraska - Lincoln

DigitalCommons@University of Nebraska - Lincoln

$11-8-2004$

\title{
Theoretical study of the magnetic ordering in rare-earth compounds with face-centered-cubic structure
}

\author{
Chun-gang Duan \\ University of Nebraska-Lincoln, cgduan@clpm.ecnu.edu.cn \\ Renat F. Sabirianov \\ University of Nebraska - Omaha, rsabirianov@mail.unomaha.edu \\ Jianjun Liu \\ University of Nebraska- Lincoln, jliu@unlserve.unl.edu \\ Wai-Ning Mei \\ University of Nebraska at Omaha, physmei@unomaha.edu \\ Peter A. Dowben \\ University of Nebraska-Lincoln, pdowben@unl.edu
}

See next page for additional authors

Follow this and additional works at: https://digitalcommons.unl.edu/physicsdowben

Part of the Physics Commons

Duan, Chun-gang; Sabirianov, Renat F.; Liu, Jianjun; Mei, Wai-Ning; Dowben, Peter A.; and Hardy, John R., "Theoretical study of the magnetic ordering in rare-earth compounds with face-centered-cubic structure" (2004). Peter Dowben Publications. 5.

https://digitalcommons.unl.edu/physicsdowben/5

This Article is brought to you for free and open access by the Research Papers in Physics and Astronomy at DigitalCommons@University of Nebraska - Lincoln. It has been accepted for inclusion in Peter Dowben Publications by an authorized administrator of DigitalCommons@University of Nebraska - Lincoln. 


\section{Authors}

Chun-gang Duan, Renat F. Sabirianov, Jianjun Liu, Wai-Ning Mei, Peter A. Dowben, and John R. Hardy 


\title{
Theoretical study of the magnetic ordering in rare-earth compounds with face-centered-cubic structure
}

\author{
Chun-gang Duan, ${ }^{\text {a) }}$ R. F. Sabiryanov, ${ }^{\text {b) }}$ Jianjun Liu, and W. N. Mei \\ Department of Physics, University of Nebraska at Omaha, Omaha, Nebraska 68182-0266 \\ P. A. Dowben and J. R. Hardy \\ Department of Physics and Center for Materials Research and Analysis, University of Nebraska \\ at Lincoln, Lincoln, Nebraska 68588
}

(Presented on 8 November 2004; published online 2 May 2005)

\begin{abstract}
We present a detailed theoretical study of the magnetic ordering in heavy rare-earth compounds with a face-centered-cubic structure. In addition to the exchange interactions which are counted up to the third nearest neighbors, the effect of the dipolar interactions and magnetic anisotropic effect are also included in our model Hamiltonian. The interactions parameters are obtained from first-principles band-structure calculations by fitting the total energies of different magnetic configurations to the Heisenberg Model. Thus from utilizing the Monte Carlo simulations, we explained the formation of different magnetic structures in the rare-earth compounds. (C) 2005 American Institute of Physics. [DOI: $10.1063 / 1.1851692]$
\end{abstract}

\section{INTRODUCTION}

The rare earths have long been an interesting and challenging subject to physicists due to their unique magnetic, electric, and optical properties. For example, they show various fascinating physical phenomena, such as magneticoptical effect, heavy-fermion state, dense Kondo effect, magnetic polaron effect, etc. In addition, they are also of great importance to the industries due to their numerous technological applications. ${ }^{1}$

The magnetic properties of the rare earths, as well as those interesting many-body phenomena, are intimately related to the highly localized $4 f$ electrons of the open shell. As early as seventy years ago, Van Vleck et al. had studied the magnetic properties of rare-earth ions with the help of the newly developed quantum mechanics. ${ }^{2}$ Their work suggested that the $4 f$ electrons responsible for the magnetism of the rare earth are sequestered in the interior of the atom, and so experience only a small crystalline field. ${ }^{3}$ Thus, they explained why Hund's rule with $L-S$ coupling could give values of the magnetic moments of rare-earth ions very close to experimental observations, at least in the high-temperature region.

However, understanding the magnetic ordering of the rare earths is still a challenging problem to the magnetists. Even for the rare-earth pnictides $R X(X=\mathrm{N}, \mathrm{P}, \mathrm{As}, \mathrm{Sb}, \mathrm{Bi})$, which have simple $\mathrm{NaCl}$-type structures, various kinds of magnetic ordering are observed. ${ }^{4-6}$ The neutron-diffraction study on powered specimens by Child et al. ${ }^{4}$ and the theoretical discussion based on empirical parameter calculation by Trammell ${ }^{7}$ enriched our knowledge of the magnetic properties of these rare-earth compounds. But only until the recent ten years, with the advance of high-quality single-

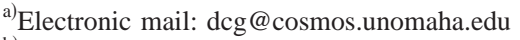

b) Electronic mali: rsabirianov@mail.unomaha.edu
}

crystal growing technique and the rapid increase of computational ability, have we been able to study the magnetic ordering from a microscopic view.

Unlike those $3 d$ ferromagnets, the rare earths generally have large magnetic moments, yet the exchange interactions between local spins are relatively weak. Thus other factors, such as magnetic dipole interactions, may also have significant influence on their magnetic orderings. In addition, the coupling between neighboring lattice sites is believed to be through an indirect magnetic exchange: Ruderman-KittelKasuya-Yosida (RKKY) type, which is essentially a longrange interaction and requires considerations of the exchange interactions between a large number of neighboring atoms. Furthermore, the magnetic orderings of face-centered-cubic (fcc) structure are much more complicated than that of a simple cubic or body-centered-cubic structure, because in the fcc structure two nearest neighboring $(\mathrm{NN})$ atoms of a given atom can themselves be NN atoms. All these factors make the problem very complex.

In this paper, we summarize our study on the magnetic ordering of $R X$ with fcc structure. We proposed a spin Hamiltonian which contains not only the exchange interaction, but also the dipolar interaction and anisotropic term. First-principles calculations are applied to two typical $R X$ compounds, i.e., ErN and ErAs, with four different magnetic orderings to deduce those interaction parameters, which were then used in our Monte Carlo (MC) simulations. In this way, we elucidated the mechanism that controls the magnetic ordering in rare-earth compounds. Finally, transition temperatures obtained from MC simulations were used to compare with the experimental values, thus gives a direct verification of our theory.

\section{MODEL HAMILTONIAN}

The magnetic transition temperatures of these rare-earth salts are generally very low. Most of them are below $20 \mathrm{~K}$, only some nitrides have relatively higher values. Hence this 
implies that the exchange interactions in these compounds are weak. Therefore, those interactions ignored in the study of transition magnetic systems should be reconsidered in cases studied in the current work. For example, the crystal structure of ErAs is fcc with a lattice constant of $5.73 \AA$, when the dipole moment is about $9.5 \mu_{B}$ (value for a free $\left.\mathrm{Er}^{3+}\right)$; the energy difference per formula between the ferromagnetic and antiferromagnetic configurations could be around $0.2 \mathrm{meV}$, which corresponds to a thermal energy of $2 \mathrm{~K}$, being comparable with the Néel temperature of $4.5 \mathrm{~K}$ of ErAs.

Due to the same reason, the exchange interactions are counted up to the third NN atoms. In a fcc structure, the ratio of the third and second nearest distances is 1.225 , which is smaller than that of the second and first nearest distances (1.414). Furthermore, considering that the number of the third NN atoms is 24 while that of the second $\mathrm{NN}$ atoms is only 6 , if the exchange parameter follows the RKKY type; $J \sim \cos \left(2 k_{F} r\right) / r^{3}$, we found that there is no convincing reason to exclude the contributions from the third NN atoms. It is reasonable to ignore the interactions with the fourth $\mathrm{NN}$ atoms since their number (12) is relatively small.

The anisotropic effect is also apparent in $R X$ compounds. According to Child et al., ${ }^{4}$ the easy axis of these compounds could have at least three directions, i.e., [001], [111], and perpendicular to [111]. This kind of anisotropy could not be easily explained in terms of dipolar interactions.

Therefore, in order to incorporate all the abovementioned effects when studying the magnetic properties of the $R X$ compounds, we introduce the following spin Hamiltonian:

$$
\begin{aligned}
H= & \sum_{n=1,2,3} J_{n} \sum_{i>j}^{n \text {th NN }}\left(\mathbf{S}_{i} \cdot \mathbf{S}_{j}\right)+\sum_{i>j} D_{i j} \\
& +K_{1} \sum_{i}\left(\alpha_{i}^{2} \beta_{i}^{2}+\beta_{i}^{2} \gamma_{i}^{2}+\gamma_{i}^{2} \alpha_{i}^{2}\right)+K_{2} \sum_{i} \alpha_{i}^{2} \beta_{i}^{2} \gamma_{i}^{2},
\end{aligned}
$$

where $\mathbf{S}_{i}$ indicates the spin vector at the $i$ th lattice site, $J_{n}$ is the exchange parameter between the $n$th neighboring atoms, the dipole-dipole interaction energy $\mathbf{D}_{i j}$ between two magnetic dipoles $\mathbf{u}_{i}$ and $\mathbf{u}_{j}$ is given by,

$$
D_{i j}=\frac{\left(\mathbf{u}_{i} \cdot \mathbf{u}_{j}\right)-\left[3\left(\mathbf{n} \cdot \mathbf{u}_{i}\right)\left(\mathbf{n} \cdot \mathbf{u}_{j}\right)\right]}{\left|\mathbf{r}_{i j}\right|^{3}},
$$

with $\mathbf{n}$ as a unit vector in the direction $\mathbf{r}_{i j} . \alpha, \beta$, and $\gamma$ are the direction cosines and $K_{1}$ and $K_{2}$ are the first- and secondorder anisotropic constants. For cubic systems, there is no $K_{0} \alpha^{2}$ term.

\section{TOTAL-ENERGY CALCULATION}

The exchange parameters in the model Hamiltonian can be obtained from the first-principles band-structure calculations. To do this, we carried out total-energy calculations on four different magnetic orderings of the fcc structure. One of them is ferromagnetic (FM) ordering as ErN has, the other three are antiferromagnetic (AFM) orderings, as shown in Ref. 8. In the first AFM ordering (AFM I), which is called face-centered first kind, the ferromagnetic sheet is in the $x$
TABLE I. Calculated total-energy differences (meV) per formula between FM and AFM configurations for each AFM structures of ErN and ErAs. $\Delta E_{n}=E_{\mathrm{FM}}-E_{\mathrm{AFM}_{n}}$. The exchange parameters $J_{1}, J_{2}$, and $J_{3}$ are then calculated from these energy differences.

\begin{tabular}{lcccrrr}
\hline \hline & $\Delta E_{1}$ & $\Delta E_{2}$ & \multicolumn{1}{c}{$\Delta E_{3}$} & \multicolumn{1}{c}{$J_{1}$} & \multicolumn{1}{c}{$J_{2}$} & \multicolumn{1}{c}{$J_{3}$} \\
\hline ErN & 0.0 & -3.8 & -0.5 & 0.192 & -0.633 & -0.096 \\
ErAs & 0.54 & 3.23 & 0.53 & -0.170 & 0.471 & 0.119 \\
\hline \hline
\end{tabular}

$-y$ (or $x-z, y-z)$ plane. CeBi was found to have this kind of AFM ordering. ${ }^{5}$ In the second AFM ordering (AFM II), facecentered second kind (type I), the ferromagnetic sheet is along the [111] plane. Most of the $R X$ compounds, including ErAs, were found to have this Mn-O-type AFM structure. The third AFM structure (AFM III) is a modification of the first one. In fact, more AFM orderings can be found in Ref. 9 , but the four structures we chose are enough for us to get the exchange parameters.

Our electronic structure calculations are based on the density-functional theory in the local spin-density approximation (LSDA) with additional Hubbard correlation terms describing on-site electron-electron repulsion associated with the $4 f$ narrow bands (LSDA $+U$ approach). ${ }^{10,11}$ The first-principles band-structure approach applied in this work is the full-potential linear-augmented-plane-wave (FLAPW) method. ${ }^{12}$ The calculated results agree well with photoemission experiments. ${ }^{13,14}$

We chose ErN and ErAs as the candidate materials to study the magnetic properties of $R X$ compounds. Muffin-tin radii of Er, N, and As atoms are set to be 2.6, 1.8, 2.2 a.u., respectively. In the total-energy calculation, the factor $R_{\mathrm{MT}} K_{\max }$ is chosen to be 8 . We found that using as large as $4000 k$ points in the Brillouin zone is necessary to obtain the energy convergence up to $0.1 \mathrm{meV}$. Following previous works, ${ }^{13,14}$ we used values for $U=8.6 \mathrm{eV}$ and $J=0.75 \mathrm{eV}$ in the LSDA $+U$ scheme.

The total exchange energies per lattice site of the four magnetic orderings can be explicitly expressed from the Heisenberg Hamiltonian [Eq. (1)] as,

$$
\begin{aligned}
& E_{\mathrm{FM}}=E_{0}+6 J_{1}+3 J_{2}+12 J_{3}, \\
& E_{\mathrm{AFM}_{\mathrm{I}}}=E_{0}-2 J_{1}+3 J_{2}-4 J_{3}, \\
& E_{\mathrm{AFM}_{\mathrm{II}}}=E_{0}-3 J_{2}, \\
& E_{\mathrm{AFM}_{\mathrm{III}}}=E_{0}-2 J_{1}+J_{2}+4 J_{3},
\end{aligned}
$$

where $E_{0}$ is the reference energy.

Due to the fact that the energy differences between these orderings are generally very small, extreme care is needed in the calculations. Hence, we only compare the energies between FM and AFM configurations of each different structure to avoid any error caused by the different symmetry or shape. These energy differences are then used to solve Eqs. (3)-(6). Considerable efforts are also needed to prevent the calculation traps into a metastable state. The final results are listed in Table I. As we can see, our LSDA $+U$ calculation gives correct ground-state AFM II for ErAs. For ErN, we 
found that the FM and the first AFM ordering can be regarded as degenerate states within the accuracy of our calculation. All the $J_{n}$ values are very small, which is expected because of the low transition temperatures.

\section{MONTE CARLO SIMULATION}

MC simulations, based on the model Hamiltonian Eqs. (1) and (2), are used to find the ground states of a fcc structure with different values of $J_{n}, \mathbf{u}_{i}, K_{1}$, and $K_{2}$. The same method has been applied successfully in the study of complex permanent magnetic materials. ${ }^{15}$

The lattice studied in our MC simulation is a $10 \times 10$ $\times 10$ fcc cell (4000 spins) with lattice constants equal to $4.83 \AA(\mathrm{ErN})$ and $5.73 \AA$ (ErAs), respectively. Periodic boundary conditions are applied. The systems are heated up to $100 \mathrm{~K}$, then are slowly quenched to $0 \mathrm{~K}$ by $30-50$ steps. In each temperature step, $3000 \mathrm{MC}$ updates per spin are carried out. The anisotropic coefficients $K_{1}$ and $K_{2}$ are set to be 0.1 and $0.05 \mathrm{meV}$ per formula for $\mathrm{ErN}$, and -0.1 and $0.5 \mathrm{meV}$ per formula for ErAs. The dipole moments in both cases are set to be $9.5 \mu_{B}$.

Generally, the direct sum of the dipole-dipole interaction term in an infinite lattice, here a fcc structure with lattice constant $a$, will most likely encounter the problem of slow numerical convergence. Usually it can be solved by a special technique such as the Ewald summation method. However, our calculation shows that the difference between the results obtained by the direct sum under a cutoff distance of $8 a$ and $100 a$ is no more than $0.5 \%$, and the latter agrees almost exactly with the value obtained from analytical method. ${ }^{16}$ Thus in our simulations, the dipolar interaction is included up to a range of $8 a$.

Contrary to the general belief that the dipolar interaction can solely account for stabilizing the magnetic ordering in a fcc structure, ${ }^{6}$ we found that in our simulation, though the fcc spin lattice with a pure dipole-dipole interaction has a ground state of AFM II type with all the dipole moments perpendicular to the [111] axis, there also exist several metastable states with complex magnetic orderings which have energies only about $10^{-4} \mathrm{meV}$ higher. Such small energy difference can easily be reached by even a tiny thermal fluctuation (around $1 \mathrm{mK}$ ), which renders the system unstable. Nevertheless, the existence of dipole interaction really contributes significant anisotropy to the AFM system like ErAs.

When all factors are taken into account, we found that, for ErAs, the ground-state magnetic configuration obtained from MC calculations agrees with experimental observations. The obtained transition temperature (from paramagnetic to AFM) is around $6 \mathrm{~K}$, which agrees well with the experimental value of $4.5 \mathrm{~K} .{ }^{6}$ For ErN, we found the ground state to be AFM. This is because of the incorrect prediction of the ground state of ErN by total-energy calculations. The cause of this is still under investigation.

\section{CONCLUSIONS}

In conclusion, we applied the first-principles calculations to the study of the magnetic properties of rare-earth compounds. The exchange parameters of these systems have been obtained directly from theoretical calculation, instead of from fitting with experimental observations. The magnetic ordering of rare-earth compounds with a face-centered-cubic structure is determined from the intriguing competition between the first, second, and third NN exchange interactions. In addition, we found that the dipole-dipole interactions could play an important role in the magnetic behaviors of the rare-earth compounds, and are especially instrumental to the magnetic anisotropy.

\section{ACKNOWLEDGMENTS}

This work was supported by the Office of Naval Research, Nebraska Research Initiative, the Nebraska-EPSCoRNSF Grant No. EPS-9720643 and Department of the Army Grant Nos. DAAG 55-98-1-0273 and DAAG 55-99-1-0106.

${ }^{1}$ Handbook on Physics and Chemistry of Rare Earth (North-Holland, Amsterdam, 1979).

${ }^{2}$ J. H. Van Vleck, The Theory of Electric and Magnetic Susceptibilities (Oxford University Press, New York, 1932), Chap. IX.

${ }^{3}$ J. H. Van Vleck, Rev. Mod. Phys. 50, 181 (1978).

${ }^{4}$ H. R. Child, M. K. Wilkinson, J. W. Cable, W. C. Koehler, and E. O. Wollan, Phys. Rev. 131, 922 (1963).

${ }^{5}$ T. Chattopadhyay, P. Burlet, J. Rosat-Mignod, H. Bartholin, C. Vettier, and O. Vogt, Phys. Rev. B 49, 15096 (1994).

${ }^{6}$ S. J. Allen, Jr., N. Tabatabaie, C. J. Palmstrøm, G. W. Hull, T. Sands, F. DeRosa, H. L. Gilchrist, and K. C. Garrison, Phys. Rev. Lett. 62, 2309 (1989).

${ }^{7}$ G. T. Trammell, Phys. Rev. 131, 932 (1963).

${ }^{8}$ J. Samuel Smart, Effective Field Theories of Magnetism (W. B. Saunders Company, Philadelphia, 1966), pp. 76-77.

${ }^{9} \mathrm{~J}$. B. Goodenough, Magnetism and the Chemical Bond (Wiley, New York, 1963), p. 92.

${ }^{10}$ V. I. Anisimov, J. Zaanen, and O. K. Andersen, Phys. Rev. B 44, 943 (1991).

${ }^{11}$ V. I. Anisimov, I. V. Solovyev, M. A. Korotin, M. T. Czyzyk, and G. A. Sawatzky, Phys. Rev. B 48, 16929 (1993).

${ }^{12}$ P. Blaha, K. Schwarz, G. K. H. Madsen, D. Kvasnicka, and J. Luitz, WIEN2k, An Augmented Plane Wave+Local Orbitals Program for Calculating Crystal Properties (Karlheinz Schwarz, Techn. Universität Wien, Austria, 2001).

${ }^{13}$ T. Komesu, H K. Jeong, J. Choi, C. N. Borca, P. A. Dowben, A. G. Petukhov, B. D. Schultz, and C. J. Palmstrøm, Phys. Rev. B 67, 035104 (2003).

${ }^{14}$ C.-G. Duan et al., Surface Review and Letters 11, No. 6 (2004).

${ }^{15}$ R. F. Sabiryanov and S. S. Jaswal, Phys. Rev. Lett. 79, 155 (1997).

${ }^{16}$ M. H. Cohen and F. Keffer, Phys. Rev. 99, 1128 (1955). 